Urologe $2022 \cdot 61: 622-628$

https://doi.org/10.1007/s00120-021-01737-9

Angenommen: 25. November 2021

Online publiziert: 17. Februar 2022

○ Der/die Autor(en) 2022

\section{Stellenwert wasserbezogener Rehabilitationsmaßnahmen bei Stomaträgern}

\section{Ergebnisse eines Pilotprojekts und Literaturübersicht}

\author{
Juliane Putz · Angelika Borkowetz - Gina Benita Schlumberger - Johannes Huber · \\ Christian Thomas \\ Klinik und Poliklinik für Urologie, Universitätsklinikum Carl Gustav Carus Dresden, Technische Universität \\ Dresden, Dresden, Deutschland
}

Hintergrund: Die Anlage eines Hautstomas nach exenterativen Beckeneingriffen stellt für Betroffene einen drastischen Einschnitt in die körperliche Integrität dar. Vielfach führen ästhetische und funktionelle Bedenken dazu, dass wasserbezogene Angebote zur Rehabilitation nicht genutzt werden.

Fragestellung: Ziel war es, im Rahmen des Pilotprojekts „UROAquaFIT“ die Möglichkeit einer wasserbezogenen Rehabilitationsmaßnahme für Patienten nach Zystektomie mit inkontinenter Harnableitung zu evaluieren.

Methode: Im Rahmen des Pilotkurses „UROAquaFIT“ wurde Patienten nach radikaler Zystektomie mit inkontinenter Harnableitung die Möglichkeit einer wasserbezogenen Rehabilitationsmaßnahme angeboten. Aufgeteilt in Kleingruppen erfolgte ein Wassergymnastikkurs unter Anleitung einer Physiotherapeutin und einer Stomatherapeutin unter ärztlicher Aufsicht. Daten zur Lebensqualität und der persönlichen Beurteilung des Kurses mittels Fragebögen wurden vor Beginn und 6 Monate nach Kursende erhoben und qualitativ ausgewertet. Darüber hinaus erfolgte eine Literaturrecherche (MEDLINE) zum Thema Aquagymnastik in der Onkologie. Ergebnis und Diskussion: Das Kursangebot wurde von allen Teilnehmern als durchweg positiv und bereichernd empfunden. Wohlbefinden und Selbstwertgefühl der Probanden konnten gesteigert werden. Wassergymnastik unter gezielter physio- und stomatherapeutischer Anleitung kann somit eine höchst effektive Rehabilitationsmaßnahme für Patienten nach inkontinenter Harnableitung darstellen. Darüber hinaus stärkt sie die körperliche Integrität der Betroffenen und erweitert somit die Möglichkeiten der sozialen Reintegration. Diese Form der Therapie sollte als fester Baustein in der Rehabilitation nach exenterativen Beckeneingriffen mit Stomaanlage verankert werden.

\title{
Schlüsselwörter
}

Conduit · Wassergymnastik · Lebensqualität · FACT-G · EORTC QLQ-30 · UROAquaFIT

\section{Einleitung}

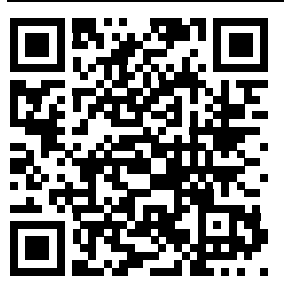

QR-Code scannen \& Beitrag online lesen
Die Anlage eines Hautstomas nach Zystektomie oder Beckenexenteration stellt einen tiefgreifenden Einschnitt für die betroffenen Patienten dar. Nicht nur die Notwendigkeit der Auseinandersetzung mit der zumeist tumorbedingten Diagnose [1], sondern auch die Veränderung des empfundenen Körperbildes führen zu einer Be- einflussung der körperlichen Integrität und oftmals auch des Selbstbildes. Darüber hinaus besteht beispielsweise nach operativer Entfernung der Harnblase und inkontinenter Harnableitung initial eine eingeschränkte körperliche Belastbarkeit, die besondere Anforderungen an rehabilitative Maßnahmen stellt [9-11, 14].

Im klinischen Alltag existieren zwar postoperative Rehabilitationsmaßnah- 
Infobox

PRAXISTIPP - Rezeptierung von Schwimmbandagen zur Stomaversorgung

Feldbefüllung Rezept:

Hilfsmittel

1 Stück xxxxx (Name Bandage)

HMV Pos.-Nr.: xxxxxx (entsprechend Bandage) Diagnose: Urothelkarzinom der Harnblase etc. Stomaart: Urostomie/lleumconduit etc. Indikation: K43.9

men zur Stärkung der physischen Fitness, jedoch erfolgen diese zumeist ohne Nutzung des Elements Wasser [13].

Auf den ersten Blick stellt die Wassergymnastik eine besondere Herausforderung für diese Patienten dar - sowohl funktionell als auch von ästhetischer Seite. Bisher gibt es keine wissenschaftlich untersuchten wasserbezogenen Trainingsmöglichkeiten für diese Patientengruppe. Dies ist bedauerlich, da sich Wassergymnastik und Schwimmen als eine optimale Maßnahme zum Muskelaufbau und zur Stärkung der Knochendichte sowie Verbesserung der kardiovaskulären Funktion anbieten [15, 16, 18].

Aus diesem Grund wurde das Pilotprojekt „UROAquaFIT" an der Klinik und Poliklinik für Urologie des Universitätsklinikums Carl Gustav Carus Dresden (UKD) initiiert. Ziel dieses Kurses war es, Patienten mit inkontinenter Harnableitung nach Zystektomie unter professioneller physiound stomatherapeutischer Anleitung die Möglichkeit einer wasserbezogenen Rehabilitationsmaßnahme zu bieten. Darüber hinaus führten wir eine Literaturrecherche zu dem Thema Wassersport und Onkologie durch.

\section{Methode}

\section{Pilotprojekt „UROAquaFIT"}

Im Rahmen der Projektförderung „Rudern gegen Krebs" des Nationalen Zentrums für Tumorerkrankungen (NCT) etablierten wir im Jahr 2019 das Pilotprojekt „UROAquaFIT“ [12]. Die Teilnahme wurde Patienten mit inkontinenter Harnableitung (Ileumconduit oder Ureterokutaneostomie) nach radikaler Zystektomie angeboten. Eingeschlossen wurden Patienten mit einem postoperativen Zeitintervall von mindestens 6 Monaten sowie mit einem guten Allgemeinzustand (ECOG 01). Tumorprogress oder laufende uroonkologische Systemtherapien waren Ausschlusskriterien. Die Kontaktaufnahme mit potenziellen Patienten erfolgte über die Zystektomiedatenbank der Klinik und Poliklinik für Urologie am Universitätsklinikum Dresden.

Die Initiierung der Wassergymnastik erfolgte unter physiotherapeutischer Anleitung über einen Zeitraum von $90 \mathrm{~min}$. Weitere 30 min wurde freies Schwimmen vollzogen. Die genaue Durchführung ist in - Tab. 1 beschrieben. In den Jahren 2019 und 2020 wurde jeweils ein Initiierungskurs angeboten. Teilnehmer des ersten Kurses konnten auch am zweiten Kurs teilnehmen.

Die Initiierungskurse basierten auf 4 Sitzungen im wöchentlichen Abstand. Im Anschluss erhielten die Patienten die Möglichkeit, die erlernten Übungen in einem heimatnahen Schwimmbad selbstständig fortzusetzen. Während der Kurse wurden die Patienten von einer Stomatherapeutin vor Ort betreut, um die Dichtigkeit des Stomabeutels zu überprüfen. Zur zusätzlichen Sicherheit wurde von den Patienten ein spezieller Schwimmgürtel, eine sog. Schwimmbandage, getragen (hier: Stomacare-Bandage HydroActive, Basko Healthcare, Zaandam, Niederlande). Alle Übungen wurden sowohl mit als auch ohne diese Bandage ausgeführt, um ggf. relevante Unterschiede zu detektieren.

Die Lebensqualität der Patienten wurde anhand von Fragebögen vor Beginn der Wassergymnastik und nach 6 Monaten evaluiert. Hierfür wurden validierte Fragebögen und selbst entwickelte Fragebögen genutzt. Als validierte Fragebögen wurden der FACT-G- (,functional assessment of cancer therapy-general") sowie der EORTC QLQ-30-Fragebogen Version 3.0 ("quality of life questionnaire" mit 30 Einzelfragen) angewendet. Der eigens für das Projekt „UROAquaFit" entwickelte Fragebogen ist in 0 Tab. 2 dargestellt.

Darüber hinaus erfolgte eine Literaturrecherche über MEDLINE bezüglich wasserbezogener Rehabilitationsmaßnahmen in der Onkologie. Hierfür wurden die drei Suchbegriffe "sport, water, oncology" verwendet. Berücksichtigt wurden in der Auswertung nur Originalarbeiten, die sich inhaltlich mit dem Thema Wassersport und
Wassergymnastik im Rahmen der Behandlung von onkologischen Patienten auseinandersetzten.

\section{Ergebnisse}

Insgesamt nahmen 12 Patienten an dem Kurs teil. Die Umsetzung des Projekts "UROAquaFIT" sowie die Teilnehmercharakteristika sind in - Tab. 1 aufgeführt.

Am Initiierungskurs 2019 nahmen insgesamt 9 Patienten teil; 7 davon besuchten mindestens 3 Trainingseinheiten; 2 Teilnehmer verstarben innerhalb von 6 Monaten nach Beendigung des Initiierungskurses 2019 aus onkologischen Gründen.

Am Initiierungskurs 2020 nahmen insgesamt 7 Patienten teil; 6 davon besuchten mindestens 3 Trainingseinheiten.

Bei keinem der Teilnehmer musste eine Trainingseinheit aufgrund gesundheitlicher Probleme abgebrochen werden. Gründe für das Nichtteilnehmen waren konkurrierende Termine oder akut krankheitsbedingte Absagen.

Während beider Initiierungskurse konnte keine Ablösung der Stomaplatte während der Übungen oder beim Schwimmen sowohl mit als auch ohne Bandage festgestellt werden. Schwimmbadassoziierte Harnwegsinfekte wurden nicht registriert. Bedingt durch die Coronapandemie konnte die initial geplante heimatnahe Fortsetzung der Wassergymnastik im Verlauf nicht durchgeführt und somit auch nicht evaluiert werden.

Die Rücklaufquote der Fragebögen vor Beginn des Kurses und nach 6 Monaten lag bei jeweils $100 \%$ für alle noch lebenden Teilnehmer.

Der für das Projekt entwickelte Fragebogen zu Kursbeginn ist in - Tab. 2 mit den entsprechenden Ergebnissen dargestellt. Hier spiegelt sich die Motivation der Teilnehmer sowie die subjektiv ursächlich empfundene zu geringe Unterstützung im Hinblick auf Anleitung zur körperlichen Rehabilitation gut wider. Nur 2 von $12 \mathrm{~Pa}$ tienten hatten bis zum Kursbeginn Erfahrung in Bezug auf Schwimmen mit Stoma. Hauptgründe für das bisherige Meiden von Wasser waren die Sorge vor Harnwegsinfektionen sowie die Gefahr der Ablösung des Stomabeutels.

Die initiale Erfassung der Lebensqualität der Teilnehmer über den FACT-G-Fra- 
Tab. 1 Umsetzung des Projekts "UROAquaFIT" sowie Teilnehmercharakteristika

\begin{tabular}{|c|c|c|}
\hline \multirow[t]{8}{*}{ Allgemeine Angaben } & Ort & Indoor-Schwimmbecken \\
\hline & Beckengröße & $13 \times 6 \mathrm{~m}\left(78 \mathrm{~m}^{2}\right)$ \\
\hline & Wassertiefe & $1,35 \mathrm{~m}$ \\
\hline & Frequenz und Dauer & $1 \times$ wöchentlich $120 \mathrm{~min}$ \\
\hline & Art der Übungen & $\begin{array}{l}\text { Klassische Aquagymnastik, } \\
\text { Übungen zur Koordination und } \\
\text { Kräftigung, } \\
\text { freies Schwimmen }\end{array}$ \\
\hline & Umsetzung der Übungen & $\begin{array}{l}\text { Stationär vs. in Bewegung, } \\
\text { Einzelübungen vs. Partnerübungen }\end{array}$ \\
\hline & $\begin{array}{l}\text { Zeitdauer der angeleiteten } \\
\text { Übungen }\end{array}$ & Bis $55 \mathrm{~min}$ \\
\hline & Hilfsmittel & $\begin{array}{l}\text { Schwimmbretter, -nudeln, -hanteln, } \\
\text {-bälle etc. }\end{array}$ \\
\hline \multicolumn{3}{|l|}{ Teilnehmerstruktur } \\
\hline Jahr & 2019 & 2020 \\
\hline Anzahl Teilnehmer $(n)$ & 9 & $\begin{array}{l}7 \\
\text { (davon } 4 \text { Wiederholungsteilnehmer } \\
\text { aus } 1 . \text { Kurs) }\end{array}$ \\
\hline Form der Harnableitung & Sämtlich Conduit & Sämtlich Conduit \\
\hline $\begin{array}{l}\text { Anzahl Ursache Harnab- } \\
\text { leitung maligne/benigne }\end{array}$ & $8 / 1$ & $5 / 2$ \\
\hline $\begin{array}{l}\text { Geschlechterverteilung } \\
\text { männlich/weiblich }\end{array}$ & $7 / 2$ & $4 / 3$ \\
\hline $\begin{array}{l}\text { Mittleres Alter in Jahren } \\
\text { und Interquartilsabstand }\end{array}$ & $68,2 / 16$ & $64,6 / 7$ \\
\hline $\begin{array}{l}\text { Maximale Teilnehmer pro } \\
\text { Kurs }(n)\end{array}$ & 8 & 7 \\
\hline
\end{tabular}

Tab. 2 Freie Fragen an die Teilnehmer vor Beginn des Projekts „UROAquaFIT" mit Ergebnissen (Mehrfachnennungen möglich; $n=12$ )

Was ist Ihre Motivation Hoffnung au zur Teilnahme? $\quad$ das Erlernen des Umgangs mit dem Stoma bei Schwimmen (Handling) $n=4$

Bestehen bei lhnen Vorkenntnisse im Schwimmen mit dem Stoma?

$\mathrm{Ja}$

$n=2$

Information via Internet

Welche Maßnahmen

haben Sie bisher in

Anspruch genommen, um postoperativ Ihre körperliche Leistung zu steigern?

Waren Sie mit den bisherigen Maßnahmen $n=3$ zufrieden?

\begin{tabular}{l|l|l|} 
Internet & & $\begin{array}{l}\text { Angst vor Lösen der Conduitplatte } \\
n=5, \\
\text { Hemmungen im Bereich des öf- } \\
\text { fentlichen Lebens im Umgang mit } \\
\text { Stoma } \\
n=3\end{array}$ \\
\hline $\begin{array}{l}\text { Reha-Sport } n=3 \\
\text { Gartenarbeit } \\
\begin{array}{l}\text { Walking } n=1 \\
\text { Radfahren } n=1\end{array}\end{array}$ & $\begin{array}{l}\text { Keine Maß- } \\
\text { nahmen } \\
n=4\end{array}$ & - \\
\hline $\begin{array}{l}\text { Ja } \\
n=3\end{array}$ & $\begin{array}{l}\text { Keine Anga- } \\
\text { be/Mangel } \\
\text { an Anleitung } \\
n=9\end{array}$ & \\
&
\end{tabular}

gebogen mit Erfassung von physischen, sozialen, emotionalen und funktionellen Aspekten zeigen die in $\mathbf{0}$ Tab. 3 dargestellten Ergebnisse.

Alle 10 Bögen der Probanden, welche auch das Follow-up erfüllen konnten, gingen in die Auswertung ein. Körperliches und emotionales Wohlbefinden waren hier zu Kursbeginn nachweislich am stärksten eingeschränkt.

Der erfasste Verlauf konnte in den Subskalen des körperlichen und emotionalen Wohlbefindens keine signifikante Verbesserung gegenüber dem Zeitpunkt der initialen Datenerfassung dokumentieren. Demgegenüber wurde durch die freien Fragen (s. unten) ein stark konträres, sehr positives Bild hierzu geliefert.

Die Ergebnisse der EORTC QLQ-C30 sind in - Tab. 4 abgebildet.

Auch hier konnten keine gravierenden Änderungen der Lebensqualität i. Allg. im Gegensatz zu den frei erfassten Fragen abgebildet werden.

Gerade die allgemeine Lebensqualität zeigte einen nahezu identischen Punktwert ( 23 vs. 22) zur Zeit vor Kursbeginn und im Follow-up.

Für die Parameter, welche sich nach Kursabsolvierung besserten (u.a. Müdigkeit, Übelkeit, Schlafstörungen oder Appetitmangel), konnten leider aufgrund der kleinen Fallzahl aktuell keine Signifikanzen ermittelt werden.

Einschränkend und erklärend hierfür muss vor allem gesagt werden, dass aufgrund der Coronapandemie die Möglichkeit der heimatnahen Fortsetzung von Aquagymnastik nicht gegeben war. Somit konnte aufgrund dessen keine valide Auswertung der FACT-G und EORTC-QLQC30 im Verlauf erfolgen.

\section{Hohe Patientenzufriedenheit}

Betrachtet man die Kursevaluation nach 6 Monaten, zeigt sich, wie hoch die Patientenzufriedenheit mit dem Angebot war und dass diese deutlich von der Maßnahme profitiert haben.

Als besonders positiv fiel dabei die Idee als solche und die gemeinschaftliche Aktivität auf. Außerdem wurden die Atmosphäre im Kurs, eine gute Interaktionsmöglichkeiten mit den Kursleiterinnen und der 
Tab. 3 Ergebnisse des FACT-G („functional assessment of cancer therapy-general“) initial, sowie 6 Monate nach Kursteilnahme am Projekt "UROAquaFIT" $(n=10)$

\begin{tabular}{|c|c|c|}
\hline Subskala & Zeitpunkt & \\
\hline & $\begin{array}{l}\text { Vor Kursbeginn } \\
n=10\end{array}$ & $\begin{array}{l}6 \text { Monate nach erfolgtem Kurs } \\
n=10\end{array}$ \\
\hline KWB (MW/SD) & $5,6 \pm 5,18$ & $6,3 \pm 7,28$ \\
\hline SWB (MW/SD) & $17,1 \pm 7,44$ & $19 \pm 4,64$ \\
\hline EWB (MW/SD) & $9,5 \pm 2,79$ & $8,6 \pm 4,5$ \\
\hline FWB (MW/SD) & $16 \pm 6,58$ & $15,9 \pm 6,5$ \\
\hline Summenscore (MW/SD) & $48,2 \pm 9,21$ & $50,3 \pm 5,96$ \\
\hline$p$-Wert des t-test & - & 0,58 (n.s.) \\
\hline \multicolumn{3}{|c|}{$\begin{array}{l}\text { Ergebnisse der FACT-G-Auswertungen aller Teilnehmer, welche auch das Follow-up erfüllen konnter } \\
\text { Angabe der jeweiligen Mittelwerte (MW) und Standardabweichungen (SD) } \\
\text { KWB körperliches Wohlbefinden; SWB soziales Wohlbefinden; EWB emotionales Wohlbefinden; } \\
\text { FWB funktionelles Wohlbefinden; MW Mittelwert; SD Standardabweichung }\end{array}$} \\
\hline
\end{tabular}

Tab. 4 Ergebnisse des EORTC (European Organization for Research and Treatment of Cancer) QLQ-C30 ("quality of life questionnaire") initial, sowie 6 Monate nach Kursteilnahme am Projekt "UROAquaFIT" $(n=10)$

\begin{tabular}{|c|c|c|c|}
\hline Items & $\begin{array}{l}\text { Vor Kursbeginn } \\
(n=10)\end{array}$ & $\begin{array}{l}6 \text { Monate nach erfolgtem } \\
\text { Kurs } \\
(n=10)\end{array}$ & $\begin{array}{l}\text { t-Test } \\
\text { ( } p \text {-Werte) }\end{array}$ \\
\hline $\begin{array}{l}\text { Globaler Gesundheitszustand/ } \\
\text { Lebensqualität }\end{array}$ & 23 & 22 & - \\
\hline \multicolumn{4}{|l|}{ Funktionelle Scores/Belastbarkeit } \\
\hline Körperlich & 93,6 & 92,3 & - \\
\hline Arbeit/Freizeit & 78,3 & 83,3 & 0,19 (n.s.) \\
\hline Emotional & 91,5 & 90 & - \\
\hline Konzentration & 88,3 & 86,6 & - \\
\hline Familie/Freunde & 79,2 & 75,8 & - \\
\hline \multicolumn{4}{|l|}{ Symptomscores } \\
\hline Müdigkeit & 16,3 & 14,8 & 0,35 (n.s.) \\
\hline Übelkeit/Erbrechen & 2,5 & 1,7 & 0,13 (n.s.) \\
\hline Schmerzen & 7,5 & 12,5 & - \\
\hline \multicolumn{4}{|l|}{ Single Items } \\
\hline Atemnot & 36,7 & 53,3 & - \\
\hline Schlafstörungen & 56,7 & 50 & 0,33 (n.s.) \\
\hline Appetitmangel & 10 & 6,7 & 0,31 (n.s.) \\
\hline Verstopfung & 20 & 10 & 0,17 (n.s) \\
\hline Durchfall & 16,7 & 16,7 & - \\
\hline Finanzielle Probleme & 20 & 13,3 & - \\
\hline \multicolumn{4}{|c|}{$\begin{array}{l}\text { Werte der EORTC-QLQ-C 30-Auswertungen zu Kursbeginn bzw. } 6 \text { Monate nach erfolgtem Schwimm- } \\
\text { kurs. Befragte Patientenanzahl initial } n=10 \text { (ohne im Verlauf verstorbene Patienten) sowie } 6 \text { Monate } \\
\text { nach erfolgtem Kurs } n=10 \text { (jeweils nur Erstteilnehmerbefragung). Darstellung der Werte als jeweilige } \\
\text { Mittelwerte. Mögliche Zahlenspanne } 0-100 \text { nach linearer Transformation. Hoher Score entsprechend } \\
\text { hohem Maß an Lebensqualität bzw. Belastbarkeit. Hoher Score im Bereich Symptomatik bzw. der } \\
\text { "single items" entsprechend einer starken Ausprägung der erfassten Symptome. Bei gebesserten } \\
\text { Werten nach Absolvierung des Kurses Testung der statistischen Signifikanz mittels t-Test } \\
\text { n.s. nicht signifikant }\end{array}$} \\
\hline
\end{tabular}

Abbau von allgemeinen Ängsten hervorgehoben.

Dabei ergaben sich folgende einzelne Erkenntnisse unter Berücksichtigung der freien Fragen $(n=10)$ :
- Subjektiver Benefit durch die Maßnahme:

9-mal "ja“, einmal fehlende Angabe.

- Nennung positiver Aspekte des Kurses: Wassergymnastik als solche (6-mal), gemeinschaftliche Aktivität (3-mal), Atmosphäre des Kurses (3-mal), Interaktion mit den Kursleiterinnen (3mal), Abbau der initialen Ängste im Umgang mit Wasser und Gewinnung von Selbstvertrauen (2-mal).

- Nennung möglicher Verbesserungen: einmaliger Ausfall der Gegenstromanlage im Wasser, Uhrzeit des Kurses, bei einigen wenigen Übungen subjektiv schwierige Umsetzung dieser im Wasser.

Neun der 10 Teilnehmer sahen sich bestärkt im Selbstbewusstsein zur Nutzung öffentlicher Schwimmangebote. Alle Teilnehmer gaben an, den Kurs weiterzuempfehlen. 3 Teilnehmer hatten das Schwimmen im Verlauf fest in den Alltag integrieren können, waren jedoch im Verlauf durch pandemiebedingte Auflagen in der konkreten Umsetzung wieder eingeschränkt.

\section{Literaturrecherche Wassersport nach Stomaanlage}

In der MEDLINE-basierten Recherche für Reha-Angebote mit Bezug zu Wassersport (letzter Zugriff 10.04.2021) ergaben sich unten den Suchbegriffen: "water", "sport" und "oncology" insgesamt 251 Treffer.

Acht von 251 (3,2\%) wissenschaftlichen Arbeiten evaluierten den Stellenwert wasserbasierter Rehabilitationsangebote für Patienten nach Tumorerkrankungen. Schwerpunkt hierbei bilden Patientenstudien nach Brustkrebs, wobei die größte Gruppe 68 Teilnehmerinnen umfasste. In keiner einzigen Studie wurde bisher der Stellenwert von Wassersport/ Wassergymnastik bei Stomaträgern evaluiert. In • Tab. 5 sind hierzu die Resultate der genannten Studien zusammengefasst $[2-8,17]$.

\section{Zusammenfassung und Diskussion} des Kurses als solchem: Schulnote 1 (,sehr gut ") 8-mal bzw. 2 ("gut $\left.{ }^{\prime \prime}\right)$ 2-mal, einmal fehlende Angabe.
Das Element Wasser ist bei Rehabilitationsmaßnahmen für Patienten nach operativen onkologischen Eingriffen nur unzureichend abgebildet. Die größte Datenlage 


\begin{tabular}{|c|c|c|c|c|c|c|c|c|c|}
\hline $\mid$ & 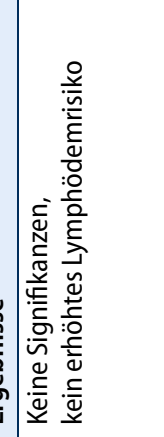 & 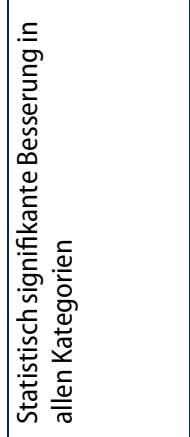 & 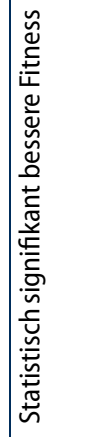 & 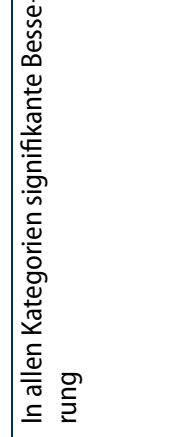 & 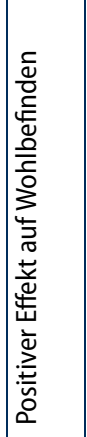 & 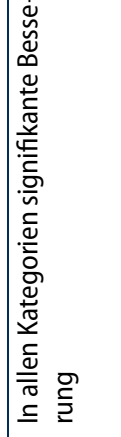 & 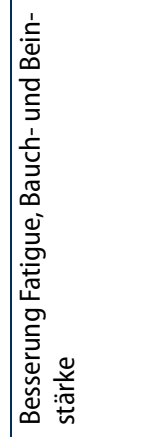 & 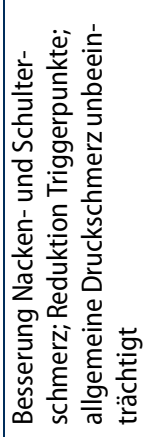 & \\
\hline 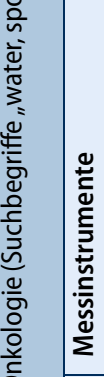 & 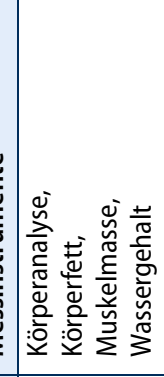 & 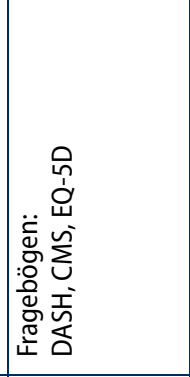 & 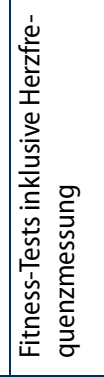 & 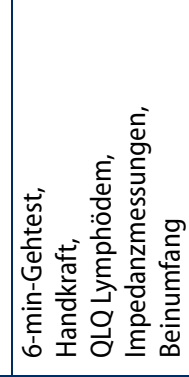 & 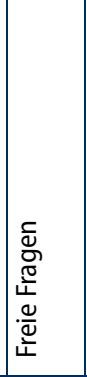 & 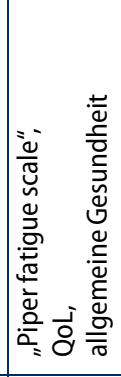 & 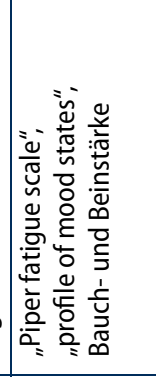 & 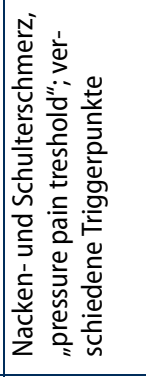 & \\
\hline 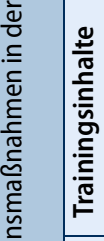 &  & 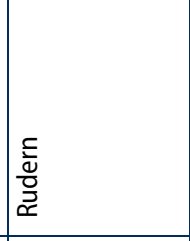 & 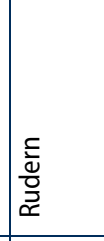 &  & 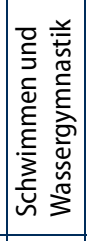 & 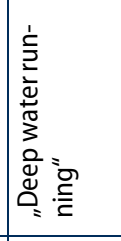 & 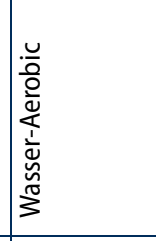 &  & \\
\hline 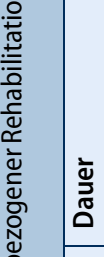 & : & 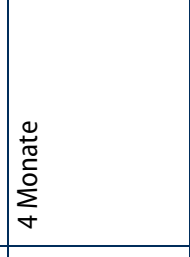 & 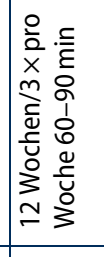 &  & 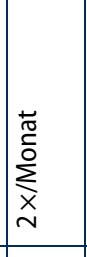 & 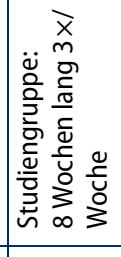 & 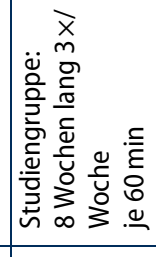 & 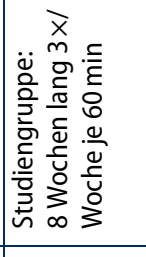 & \\
\hline  & $\begin{array}{ll}3 & 0 \\
3 & 11 \\
2 & 0\end{array}$ &  & 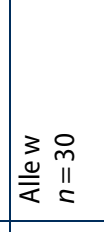 & $\begin{array}{l}3 \\
\frac{3}{\bar{\alpha}}\end{array}$ & $\underset{I}{I}$ & $\begin{array}{l}\text { F } \\
\text { II } \\
\end{array}$ & $\begin{array}{l}\infty \\
\stackrel{\infty}{0} \\
\text { II }\end{array}$ & $\begin{array}{l}\stackrel{8}{1} \\
\text { II } \\
= \\
\end{array}$ & \\
\hline 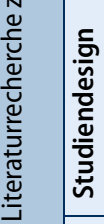 & 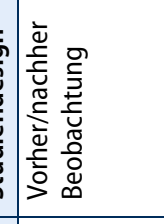 & 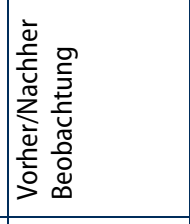 & 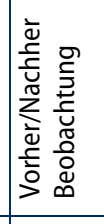 & 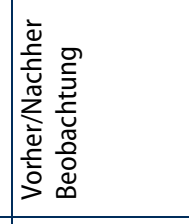 & 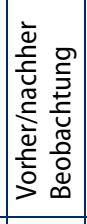 & 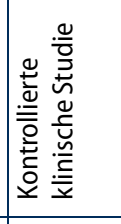 & 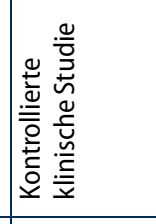 & 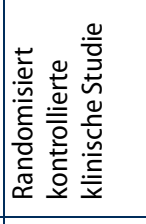 & है \\
\hline 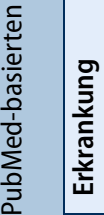 & 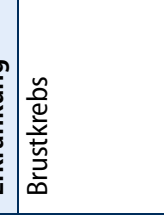 & 旁 & 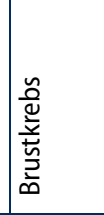 & 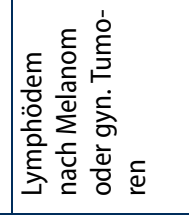 & 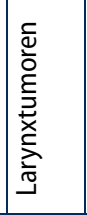 & 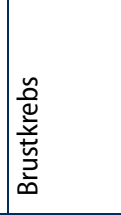 & 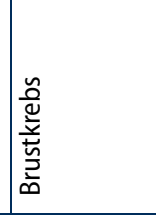 & 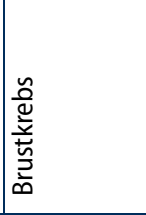 & \\
\hline 产 & $\overline{\widetilde{N}}$ & $\overline{\tilde{N}}$ & పิ & $\stackrel{\infty}{\grave{n}}$ & ¿̊ & $\stackrel{ \pm}{\grave{n}}$ & ⿳亠丷⿵冂丶 & స̃ & \\
\hline 琼 & 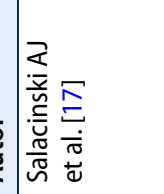 & 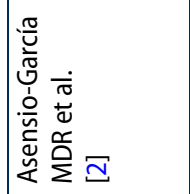 & 紊离 & 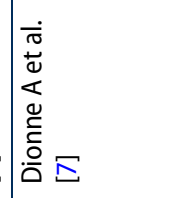 & 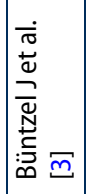 & 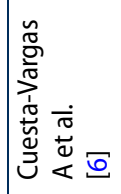 & 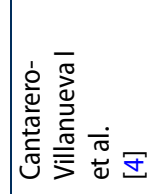 & 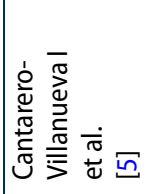 & \\
\hline
\end{tabular}


existiert für Brustkrebspatientinnen. Hier zeigt sich, dass durch die Wassergymnastik eine signifikante Verbesserung der körperlichen Fitness und der Lebensqualität erreicht werden kann. Ein entsprechend positiver Effekt durch solche Maßnahmen sollte sich, in Anlehnung an die vorhandenen Daten, entsprechend einer Hypothesengenerierung der Übertragung solcher Rehabilitationsmodelle, auch für uroonkologische Erkrankungen zeigen. Allerdings konnten wir in unserer Literaturrecherche keine Daten für Stomaträger identifizieren. Dies erklärt, weshalb sowohl von ärztlicher als auch von Patientenseite oft Unkenntnis über die Möglichkeiten der Nutzung dieser Form der körperlichen Betätigung besteht. Das durch den mutilierenden Eingriff häufig erhöhte Schamgefühl führt weiterhin dazu, dass das Element Wasser zur körperlichen Ertüchtigung von den Betroffenen kaum genutzt wird. Dies findet durch unseren freien Fragebogen vor Kursbeginn Bestätigung. Nur 2 von 12 Teilnehmern wiesen bisherige Erfahrungen in Bezug auf das Schwimmen mit Stoma auf.

Verstärkt wird dieser Informationsmangel durch fehlende Erfahrung auf ärztlicher Seite bzgl. wasserbasierter Rehabilitationsmaßnahmen. Die Option der Rezeptierung eines speziellen Schwimmgürtels zur Stomaabdeckung ist vielen ärztlichen Kolleginnen und Kollegen nicht bekannt (Empfehlung hierzu s. Infobox).

Mit einer speziell für die oben genannte Patientengruppe etablierten Maßnahme konnte in dem hier dargestellten Kursmodell "UROAquaFIT" eine Anleitung zur Aquagymnastik und zum Schwimmen mit Urostoma umgesetzt werden.

In Kleingruppen wurde den Patienten unter physiotherapeutischer Anleitung die Möglichkeit gegeben, verschiedene Bewegungsformen im Wasser auszuprobieren. Die parallele stomatherapeutische Begleitung schaffte unter diesen Bedingungen Vertrauen im Umgang mit der Harnableitung.

Wie sich in der Erfassung der Zufriedenheit mit der Maßnahme insbesondere in den freien Fragebögen zeigte, trugen die Kurse erheblich zum Abbau von Vorbehalten, Ängsten und Selbstzweifeln bei den Betroffenen bei. Auch wenn aufgrund der Coronapandemie eine heimatnahe eigeninitiativ getragene Fortsetzung der Re- habilitationsmaßnahmen nicht stattfinden konnte, gehen wir davon aus, dass bei Fortsetzung der erlernten Trainingsinhalte mit einer Steigerung der allgemeinen körperlichen Fitness und Beweglichkeit sowie Muskelaufbau und Konditionsverbesserung zu rechnen ist.

Die aktuell beschriebene kleine Fallzahl mit limitiertem Follow-up ist in Bezug auf eine valide Erhebung von Lebensqualitätsdaten noch stark eingeschränkt zu betrachten und muss für die Zukunft anhand größerer Teilnehmerzahlen verifiziert werden.

Die zahlreichen Anfragen zur Wiederholung des Angebots und die bewegenden Momente mit den Patienten nach Abschluss des Kurses lassen jedoch ein weitaus positiveres Bild vermuten, als über die oben genannten derzeitigen Messinstrumente erfasst werden konnte.

Für die in bestimmten Items nachweisbaren Vorteile konnte aufgrund kleiner Gruppengröße und den oben genannten einschränkenden Gründen derzeit noch keine Signifikanz abgeleitet werden. Der Effekt der Wassergymnastik auf die Lebensqualität und körperliche Fitness anhand validierter Fragebögen wird zeitnah in einer prospektiv angelegten Studie evaluiert. Dabei gehen wir davon aus, dass unsere hier beschriebene Hypothese des Benefits von Wassergymnastik bei Patienten mit urolonkologischen Erkrankungen und Stomaanlage untermauert werden kann.

\section{Zukunftsvision urologischer Rehabilitation nach Stomaanlage}

Ziel für die Zukunft soll es sein, das beschriebene Pilotprojekt als prospektive Studie und möglichst flächendeckend für Patientengruppen anbieten zu können. Hier könnte gerade von urologischer Seite ein längst überfälliger Beitrag zum physischen und auch psychischen Wohlbefinden unserer Patienten nach inkontinenter Harnableitung geleistet werden. Die insgesamt geringe Zahl der recherchierten wasserbezogenen Angebote für onkologische Patienten unterstreicht den Bedarf hierfür deutlich.

Angestrebt wird daher eine langfristige Kooperation mit Reha-Einrichtungen, Krankenkassen, Verbänden von Physiotherapeuten und Stomatherapeuten, um die sehr motivierenden Erfahrungen die- ses Projekts zukünftig möglichst vielen Betroffenen als Teil der Routineversorgung anbieten zu können.

\section{Fazit für die Praxis}

- Wasserbasierte Rehabilitationsmaßnahmen nach onkologischen Eingriffen sind wissenschaftlich unterrepräsentiert und werden im klinischen Alltag aktuell kaum angewendet.

- Die Durchführbarkeit und der Effekt von Wassergymnastik bei Stomaträgern nach Zystektomie und Beckenexenteration wurde bisher wissenschaftlich nicht untersucht.

- Wassergymnastik ermöglicht langfristig einen schonenden Muskelaufbau und fördert die Kondition von Patienten nach exenterativen Beckeneingriffen.

- Unter gezielter physio- und stomatherapeutischer Anleitung konnte der neu etablierte Spezialkurs "UROAquaFIT“ in Dresden die Lebensqualität und das Selbstwertgefühl betroffener Patienten steigern.

- Wasserbezogene Rehabilitationsmaßnahmen nach Beckenexenteration mit Stomaanlage sollten aufgrund des absehbar deutlichen Benefits für die Betroffenen breitflächig im Rahmen der rehabilitativen Frühförderung angeboten werden.

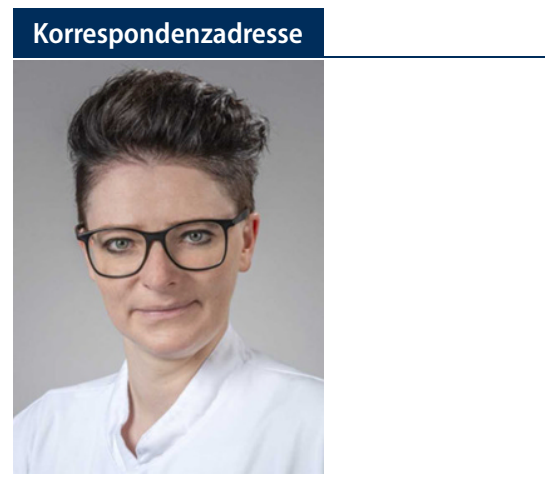

Dr. med. Juliane Putz

Klinik und Poliklinik für Urologie, Universitätsklinikum Carl Gustav Carus Dresden, Technische Universität Dresden

Fetscherstraße 74, 01307 Dresden, Deutschland juliane.putz@uniklinikum-dresden.de

Danksagung. Wir danken Frau llena Trepte vom Universitätsphysiotherapiezentrum des Universitätsklinikums Carl Gustav Carus Dresden für die physiotherapeutische und Frau Birgith Kalkbrenner für die stomatherapeutische Unterstützung. Darüber hinaus danken wir Frau Marianne Gottburg für die administrative Unterstützung. 
Förderung. Das Projekt „UROAquaFIT“ wurde von der Stiftung "Rudern gegen Krebs" außerhalb einer Studie finanziell unterstützt.

Funding. Open Access funding enabled and organized by Projekt DEAL.

\section{Einhaltung ethischer Richtlinien}

Interessenkonflikt. J. Putz, A. Borkowetz, G.B. Schlumberger, J. Huber und C. Thomas geben an, dass kein Interessenkonflikt besteht.

Für diesen Beitrag wurden von den Autoren keine Studien an Menschen oder Tieren durchgeführt. Für die aufgeführten Studien gelten die jeweils dort angegebenen ethischen Richtlinien.

Open Access. Dieser Artikel wird unter der Creative Commons Namensnennung 4.0 International Lizenz veröffentlicht, welche die Nutzung, Vervielfältigung, Bearbeitung, Verbreitung und Wiedergabe in jeglichem Medium und Format erlaubt, sofern Sie den/die ursprünglichen Autor(en) und die Quelle ordnungsgemäß nennen, einen Link zur Creative Commons Lizenz beifügen und angeben, ob Änderungen vorgenommen wurden.

Die in diesem Artikel enthaltenen Bilder und sonstiges Drittmaterial unterliegen ebenfalls der genannten Creative Commons Lizenz, sofern sich aus der Abbildungslegende nichts anderes ergibt. Sofern das betreffende Material nicht unter der genannten Creative Commons Lizenz steht und die betreffende Handlung nicht nach gesetzlichen Vorschriften erlaubt ist, ist für die oben aufgeführten Weiterverwendungen des Materials die Einwilligung des jeweiligen Rechteinhabers einzuholen.

Weitere Details zur Lizenz entnehmen Sie bitte der Lizenzinformation auf http://creativecommons.org/ licenses/by/4.0/deed.de.

\section{Literatur}

1. Antoni S, Ferlay J, Soerjomataram I et al (2017) Bladder cancer incidence and mortality: a global overview and recent trends. Eur Urol 71(1):96-108. https://doi.org/10.1016/j.eururo.2016.06.010

2. Asensio-García MDR, Tomás-Rodríguez MI, Palazón-Bru A et al (2021) Effect of rowing on mobility, functionality, and quality of life in women with and without breast cancer: a 4-month intervention. Support Care Cancer 29(5):2639-2644. https://doi. org/10.1007/s00520-020-05757-7

3. Büntzel J, Büntzel $H$, Mücke $R$ et al (2016) Sport in the rehabilitation of patients after total laryngectomy. Anticancer Res 36(6):3191-3194

4. Cantarero-Villanueva I, Fernández-Lao C, CuestaVargas Al et al (2013) The effectiveness of a deep water aquatic exercise program in cancer-related fatigue in breast cancer survivors: a randomized controlled trial. Arch Phys Med Rehabil 94(2):221-230. https://doi.org/10.1016/j. apmr.2012.09.008

5. Cantarero-Villanueva I, Fernández-Lao C, Fernández-de-Las-Peñas C et al (2012) Effectiveness of water physical therapy on pain, pressure pain sensitivity, and myofascial trigger points in breast cancer survivors: a randomized, controlled clinical

Relevance of water-related rehabilitation measures for stoma patients. Results of a pilot project and review of the literature

Background: The creation of a skin stoma after exenterative pelvic surgery drastically affects the physical health of patients. Being concerned with aesthetic and functional aspects, patients tend to not participate in offers for water-related rehabilitation, especially water gymnastics and swimming.

Objective: The aim of the pilot project "UROAquaFIT" was to evaluate the feasibility of water-related rehabilitation for patients after cystectomy with incontinent urinary diversion.

Methods: Patients after radical cystectomy with incontinent urinary diversion were offered the possibility to participate in the water-based rehabilitation program "UROAquaFIT". Split into small groups, a water gymnastics course took place under the guidance of a physiotherapist and a stoma therapist as well as under medical supervision. Data on quality of life and a personal assessment were collected using a questionnaire before and 6 months after the course. In addition, a MEDLINE-based search for aqua gymnastics in oncology was conducted.

Results and conclusion: All participants reported the course as being positive and enriching. The participants' well-being and self-esteem improved. Therefore, water gymnastics can be a highly effective method for rehabilitation of patients with incontinent urinary diversion. In addition, it improves the physical health of those affected and thus expands the possibilities for social reintegration. This form of therapy should be standard in the rehabilitation of patients with incontinent urinary diversion.

\section{Keywords}

Conduit · Water gymnastics · Quality of life · FACT-G · EORTC QLQ-30 · UROAquaFIT

trial. Pain Med 13(11):1509-1519. https://doi.org/ 10.1111/j.1526-4637.2012.01481.x

6. Cuesta-Vargas Al, Buchan J, Arroyo-Morales M (2014) A multimodal physiotherapy programme plus deep water running for improving cancerrelated fatigue and quality of life in breast cancer survivors. Eur J Cancer Care 23(1):15-21. https:// doi.org/10.1111/ecc.12114

7. Dionne A, Goulet S, Leone M et al (2018) Aquatic exercise training outcomes on functional capacity, quality of life, and lower limb lymphedema: pilot study. J Altern Complement Med 24(9-10):1007-1009. https://doi.org/10.1089/ acm.2018.0041

8. Gavala-González J, Gálvez-Fernández I, MercadéMelé Pet al (2020) Rowing training in breast cancer survivors: a longitudinal study of physical fitness. Int J Environ Res Public Health 17(14):4938. https:// doi.org/10.3390/ijerph17144938

9. Harrison JD, Young JM, Price MA et al (2009) What are the unmet supportive care needs of people with cancer? A systematic review. Support Care Cancer 17(8):1117-1128.https://doi.org/10.1007/ s00520-009-0615-5

10. Hautmann RE, Abol-Enein $\mathrm{H}$, Hafez $\mathrm{K}$ et al (2007) Urinary diversion. Urology 69(1):17-49. https:// doi.org/10.1016/j.urology.2006.05.058

11. Hedgepeth RC, Gilbert SM, He C et al (2010) Body image and bladder cancer specific quality of life in patients with ileal conduit and neobladder urinary diversions. Urology 76(3):671-675. https://doi. org/10.1016/j.urology.2010.01.087

12. Rudern gegen Krebs (2021) Webpräsenz. http:// www.rudern-gegen-krebs.de. Zugegriffen: 26. Aug. 2021

13. Jensen BT, Jensen JB, Laustsen S et al (2014) Multidisciplinary rehabilitation can impact on health-related quality of life outcome in radical cystectomy: secondary reported outcome of a randomized controlled trial. J Multidiscip Healthc 7:301-311. https://doi.org/10.2147/ JMDH.S62172

14. Modh RA, Mulhall JP, Gilbert SM (2014) Sexual dysfunction following cystectomy and urinary diversion. Nat Rev Urol 11(8):445-453. https://doi. org/10.1038/nrurol.2014.151

15. Porserud A, SherifA, TollbackA (2014) The effects of a physical exercise program after radical cystectomy for urinary bladder cancer. A pilot randomized controlled trail. Clin Rehabil 28(5):451-459. https://doi.org/10.1177/0269215513506230

16. Rammant E, Bultijnck R, Sundahl N et al (2017) Rehabilitation interventions to improve patientreported outcomesand physical fitnessinsurvivors of muscle invasive bladder cancer: a systematic review protocol. BMJ Open 7:e16054. https://doi. org/10.1136/bmjopen-2017-016054

17. Salacinski AJ, Doyle EJ, Damon Retal (2021) Effects of 12 weeks of water aerobics on body composition in those affected by breast cancer. Support Care Cancer 29(3):1205-1212. https://doi.org/10.1007/ s00520-020-05596-6

18. Teffaha D, Mourot $L$, Vernochet $P$ et al (2011) Relevance of water gymnastics in rehabilitation programs in patients with chronic heart failure or coronary disease with normal left ventricular function. J Card Fail 17(8):676-683. https://doi. org/10.1016/j.cardfail.2011.04.008 